\title{
B. Sammelberichte.
}

\section{Über die Tätigkeit der Atemzentren bei den Fischen.}

Von

\author{
Prof. Dr. Eward Babák (Prag).
}

Die vielumstrittene Frage über die Atemzentrentätigkeit der Fische scheint heutzutage schon weitgehend geklärt zu sein. Ich will es versuchen, in dieser kurzen Ábhandlung den heutigen Stand des Problems zu skizzieren und auf Grund einiger unserer neuen Untersuchungen weiter zu beleuchten.

Vor einigen Jahren haben Schoenlein und Willem ${ }^{1}$ ), und im Anschluß an sie, aber auf Grund eigener methodisch ganz neuen Forschungen Bethe $^{2}$ ) (insgesamt auf Grund von Versuchen an Haien) die Behauptung aufgestellt, daß die Atembewegungen der Fische ausschließlich auf reflektorischem Wege zustandekommen, so daß die Fische ganz wesentlich von den sämtlichen übrigen Wirbeltieren (oder wenigstens [Bethe] von den Reptilien, Vögeln und Säugetieren) sich in dieser Hinsicht unterscheiden: denn bei den letzteren geschieht die Regulierung des Atemrhythmus auf der ersten Stelle durch zentrale d. h. Blut-(Ernährungs-)Reize. Es schien, daß die völlig abweichenden ökologischen Lebensverhältnisse bei den Fischen, zugleich mit den vielen wesentlich abweichenden morphologischen und physiologischen Unterschieden einen hinreichenden Grund für ihre Sonderstellung auch in betreff der zentralen Atemmechanismen abgeben.

Im speziellen wollten Schönlein und Willem in den durch die "Konfiguration der Atemwerkzeuge " am Ende der Inspiration resp. Exspiration hervorgebrachten peripherischen Reizen (d. h. also Lage- ev. Bewegungsreizen) die Ursache der nachfolgenden Exspiration resp. Inspiration erblicken; wir müssen allerdings bemerken, daß die experimentellen Ergebnisse der Autoren zu dieser so allgemein und absolut gemachten Aussage keine ausreichende Stütze geboten haben. Demgegenüber sah Bethe in dem die Schleimhäute der Atemwege berührenden Wasser einen kontinuierlich wirkenden Reiz, welcher die - seinen Versuchsergebnissen nach vermeint-

1) Schoenlein, K. und Willem, V., Beobachtungen über Blutkreislauf und Respiration bei einigen Fischen. Zeitschr. f. Biol. 1895, Bd. XXXII, S. 590.

2) Bethe, A., Allgemeine Anatomio und Physiologie des Nervensystems. Leipzig 1903. S. $393 \mathrm{ff}$. 
lich fortfallenden - Blutreize ersetzen soll; zugleich aber kämen nach ihm auch noch die rhythmischen Reize in Betracht, welche ebenfalls peripher durch die Atembewegungen selbst hevorgebracht werden.

Diese Arbeiten und Ansichten haben einen kräftigen AnstoB zu einer Reihe von Untersuchungen ergeben, deren Ergebnisse die ganze Frage in anderes Licht gestellt haben.

Insbesondere durch Ishihara ${ }^{1}$ ) (Selachier, Teleostier) and Westerlund ${ }^{2}$ ) (Carassius), s. auch van Rynberk ${ }^{8}$ ) wurde den anscheinend so beweiskräftigen Betheschen Kokainversuchen der Boden entnommen. Bethe wollte durch vollständige Anästhesirung der Kiemen mittelst Kokain bei Erhaltung sonstiger Reflexreaktionen die Atembewegungen zum Stillstande gebracht haben; die zuletzt erwähnten Autoren haben aber gezeigt, daß auch bei vollständiger Anästhesie der Kiemen und Atemwege überhaupt die Atembewegungen weiter vor sich gehen können; der Stillstand der Atmung würde erst durch zentrale Kokainvergiftung zugeführt werden. Gleichzeitig wurde wahrscheinlich gemacht, daß es Schoenlein und Willem sowie Bethe deshalb nicht gelungen ist, durch Sauerstoffmangel des Atemwassers geeignete regulatorische Dyspnoëerscheinungen (Vermehrung und Vergrößerung der Atembewegungen) auszulösen, weil sie an frisch gefangenen, überhaupt anormalen, weitgehend schon dyspnoischen und erschöpften Fischen experimentiert hatten. Ishihara und insbesondere auch Westerlund haben nachgewiesen, daß (wenigstens) der Sauerstoffmangel zentral, durch Blutreizung der Atemzentren, eine wahre Dyspnoë auslösen kann. Dies gilt nach Baglioni s ${ }^{4}$ ) Versuchen für Fische überhaupt. Bei den mit akzessorischer Atemtätigkeit ausgestatteten Fischen, welche Babák und $D$ ědek $\left.{ }^{5}, 9\right)$ als von der Natur aus zur Entscheidung der Frage geeignete Objekte, zur Untersuchung herangezogen haben (Cobitis s. Misgurnus fossilis, Cobitis taenia mit der Darmatmung, Labyrinthfische mit der Luftatmung vermittelst der Labyrinthapparate der Kiemenhöhle), konnte die dyspnoëerregende Einwirkung des Sauerstoffmangels und der apnoëbringende EinfluB des Sauerstoffüberschusses im Blute mit aller wünschenswerten Deutlichkeit demonstriert werden; aber auch an einer Anzahl von Süßwasserteleostiern mit der alleinigen Kiemenatmung haben sie ganz auffällige dyspnoische und apnoische Zustände als vom Sauer-

1) Ishihara, M., Bemerkungen über die Atmung der Fische. Zentralbl. $f$. Pbysiol. 1906, Bd. XX, S. 157.

2) Westerlund, A., Studien über die Atembewegungen der Karausche mit besonderer Rücksicht auf den verschiedenen Gasgehalt des Atemwassers. Skand. Arch. f. Physiol. 1906, Bd. XVIII, S. 261.

a) Rynberk, G, van, Recherches sur la respiration des poissons. Arch. ital. de biol. 1906, Bd. XLV, S. 195.

4) Baglioni, S., Der Atmungsmechanismus der Fische. Ein Beitrag zur vergleichenden Physiologie des Atemrhythmus. Zeitschr, f. allg. Physiol. 1907, Bd. VIr, S. 177.

5) Babák, E., Zur Frage über das Zustandekommen der Atembewegungen bei Fischen. Nach den in Gemeinschaft mit B. Dedek durchgeführten Versuchen. Zentralbl. f. Physiol. 1907, Bd. XXI, S. 5.

6) Babák, E. und Dèdek, B., Untersuchungen über den Auslösungsreiz der Atembewegungen bei SüBwasserfischen. Pflüg. Arch. f. d. ges. Physiol, 1907, Bd. CXIX, S. 483 . 
stoffgehalte des Blutes abhängig nachgewiesen. Dies hat weiter ReuB $B^{1}$ für die Apnoë, sowie Winterstein ${ }^{2}$ ) für die Dyspnoë bestätigt.

Schwieriger haben sich die Verhältnisse der Durchforschung der zentralen Beeinflussung des Atemrhythmus durch die Kohlensäure gestaltet. Die ersten Forscher haben wohl mit zu großen Kohlendioxydmengen im äußeren Medium manipuliert, weswegen sie nur reflektorische Inhibitionsoder auch zentrale Narkosewirkungen erzielt haben. Kuiper ${ }^{8}$ ) erst hat von ganz kleinen Kohlendioxydmengen angefangen und dieselben dann vergröBert: es hat sich da keine so deutliche zentrale Dyspnoë gezeigt, wie sie sich bei den Homoiothermen schon durch ganz kleine Erhöhung des Kohlensäuregehaltes im Blute hervorbringen läßt (so daß man da oft sogar geneigt ist, die Kohlensäure für den eigentlichen Atemreiz zu halten). Die von Kuljabko ${ }^{4}$ ) verwendete Methode - durch künstliche Zirkulation einer kohlensäurehaltigen Flüssigkeit die Atemzentren zu speisen - ist an sich völlig geeignet die Frage für die Fische zu lösen, aber des Autors Ergebnisse (an abgetrennten Köpfen von Petromyzon, Acipenser, Perca, Carassius, Esox, Idus u. a. gewonnen), scheinen uns hierzu unzulänglich zu sein (es handelt sich da, wenn man die Kurvenabbildungen eingehend betrachtet, doch nicht um eine typische Dyspnoë, ähnlich derienigen der Homoiothermen). Babák und Dědek ließen die Fische die mit Kohlendioxyd geschwängerte Luft durch den Darm (Cobitis) oder die Labyrinthapparate aufnehmen; bei den letzteren Fischen sprechen die Ergebnisse eher für eine reflektorische Reizung der empfindlichen Schleimhäute; aus den Cobitisversuchen könnte man aber mit großer Wahrscheinlichkeit schließen, daß die vergrößerte Menge des Kohlendioxyds im Blute keine dyspnoischen Kiemenatembewegungen auslöst (selbst bei großem Gehalte der Kohlensäure in der verschluckten Luft hat sich das Tier normal verhalten, solange genügend Sauerstoff darin enthalten war). Später glaubt allerdings ReuB bei den Fischen (Forellen) ähnliche zentrale Wirkungen durch Kohlendioxyd veranlaßt $\mathrm{zu}$ haben, wie solche bei den Homoiothermen bekannt sind, doch ist es auch in seinen Versuchen schwierig, die zentrale und periphere Einwirkung streng auseinanderzuhalten; so z. B. beweisen seine Veronalversuche für unsere Frage gar nichts, denn wenn bei den narkotisierten Fischen nach Ubertragung in kohlendioxydhaltiges Wasser "sofort" dyspnoische Atmung einsetzte, so zeigt eben dieses "sofortige" Erscheinen der "Dyspnoë“, daß es sich höchstwahrscheinlich doch nur um reflektorische Beeinflussung handelt (die chemische Reizbarkeit kann ja die geprüfte mechanische überdauern); von größerer Bedeutung sind seine übrigen Versuche, wo bei Durchlüftung des Wassers mit einem bestimmten Sauerstoff-Kohlendioxydgemisch nach Verschwinden der anfänglichen, sichtlich reflektorisch bedingten Unruhe

1) ReuB, H., Die Wirkung der Kohlensäure auf die Atmung der niederen Wirbeltiere, insbes. d, Fische. 1. Abt. Ergebnisse der Versuche an der Regenbogenforelle. Zeitschr. f. Biol, 1911, Bd. LIII, S. 555.

2) Winterstein, H., Beiträge zur Kenntnis der Fischatmung, Pflüg. Arch. f. d. ges. Physiol. 1908, Bd. CXXV, S. 73.

3) Kuiper. T., Untersuchungen über die Atmung der Teleostier. ibid. 1907, Bd. CXVII, S. 1.

4) Kuljabko, A., Quelques expériences sur la survie prolongée de la tête isolée des poissons. Arch. internat. de physiol. 1907, Bd. IV, S. 437. 
sehr lange eine ausgesprochene Dyspnoë sich erhält, ehe die Lähmungssymptome sich entwickeln.

Während also die Frage über die dyspnoëerregende Wirkung der Kohlendioxydanhäufung im Blute uns noch weitere Nachforschungen zu erfordern scheint, insbesondere vermittelst der künstlichen Durchspülung des Zentralnervensystems, ist eine solche Wirkung des Sauerstoffmangels kaum zu bezweifeln. Einige Unklarheiten bleiben aber trotzdem bestehen; so z. B. gibt Kuiper an, daß durch seine analytischen Versuche (reiner Sauerstoffmangel, reiner KohlensäureüberschuB) das Verhalten der Fische im „altwerdenden "Wasser (wo die Fisehe hermetisch verschlossen waren) keine ausreichende Erklärung erheischt: er hat nämlich in diesem Falle eine stetige Abnahme der Atemexkursionen bei zuerst größerer, später kleinerer Frequenz beobachtet, während $\mathrm{er}$ in den analytischen Versuchen eine progressive $\mathrm{Ab}$ nahme sowohl der Höhe als auch der Frequenz gesehen hatte. Ohne auf sein Bemühen den Grund dafür durch Erwägung zu erschließen einzugehen, bemerken wir nur, daB es eigentlich unzutreffend ist, wenn er die Ergebnisse seiner lange protrahierten Versuche mit „alterndem" Wasser mit denjenigen der kurzdauernden Sauerstoffmangel- und Kohlensäureexperimente vergleicht. In einer anderen Hinsicht können wir aber $\mathrm{K}$ uiper zastimmen, daß nämlich (insbesondere in den Temperaturversuchen) die Exkursionshöhe und die Frequenz der Kiemenatembewegungen keinesfalls immer parallel sich ändern. Er führt weiter aus: die Abnahme der Exkursionen könnte man als Folge einer Abnahme der allgemeinen Erregbarkeit auffassen, während die Anderungen der Atemfrequenz auf eigentliche Reizwirkungen auslaufen würden. Es läßt sich nicht beurteilen, bis zu welchem Maße diese Auffassung eine Berechtigung haben könnte. - Noch andere Unklarheiten der Versuchsergebnisse könnte jch anführen, aber ich will davon Abstand nehmen umsomehr, da es mir vielleicht gelingen wird, später einmal auf sie einzugehen zugleich mit einer Erklärung, bis die diesbezüglichen experimentellen Untersuchungen beendet sein werden. -

Auf Grund der Versuche von Ishihara, Westerlund, Kuiper, Baglioni und Lombroso ${ }^{1}$ ) läßt sich mit Bestimmtheit behaupten, daß für das Zustandekommen des Atemrhythmus die mechanische Berührung der Atemwege und Kiemen mit Wasser keineswegs notwendig ist, während dieselbe allerdings unter den normalen Bedingungen einen wichtigen Hilfsfaktor der Atemregulation darstellt. Weit größere Bedeutung für die Regulation der Atembewegungen scheint aber den in neren Reizen zuzukommen, wie solche eben in den sich bewegenden Teilen des Atemapparates ausgelöst werden. - Deganello ${ }^{2}$ ) hat wichtige Aufschlüsse über die Rolle des Vagus und Trigeminus gemacht; es sind weiter auch Lombrosos ${ }^{3}$ ) Versuchsan-

1) Lombroso, N., Uber den Ursprung der Atembewegungen der Fische. Die Bedeutung des physikalischen Mediums. Pflüg. Arch. f. d. ges. Physiol, 1908. Bd. CXXV. S. 163.

2) Deganello, U., Die peripherischen nervösen Apparate des Atemrhythmus bei Knochenfischen, Eine anatomische und physiologische Untersuchung. Ibid. 1908, Bd. CXXIII, S. 40.

3) Lombroso, N., Uber einige besondere Regulationsvorgänge der Atembewegungen bei Knochenfischen. Ihid. 1907, Bd. CXIX, S. 1. 
stellungen hervorzuheben (über gewisse „Kompensations "-Beziehungen zwischen der Tätigkeit der Mundöffnung und der Kiemendeckelspalten).

Nach Schoenlein und Willem sowie Bethe (s. oben) hat neuerdings insbesondere Baglioni diese "reflektorische Automatie" als Selbststeuerung der Atmung mit Nachdruck betont (allerdings werden im Gegensatze zu der von Hering und Breuer angenommenen Selbststeuerung bei den Säugetieren - vermittelst der sensiblen Nerven der Atemoberflächen die sensiblen Atemmuskelnerven in den Vordergrund gestellt). Baglioni sieht insbesondere in gewissen "Ausspeireflexen" eine indirekte Stütze für diese Ansicht, indem z. B. zur Entfernung eines Fremdkörpers aus den Atemwegen zuerst eine mächtige Inspiration und dann erst eine starke Exspiration durch den geöffneten Mund vollführt wird, „als ob eben zum Zustandekommen eines heftigen Expirationsaktes noch die adäquaten peripherischen Reize eines übertriebenen Inspirationsaktes notwendig wären". Direkte Beweise dieser "reflektorischen Automatie (daB durch die Exspiration die folgende Inspiration, durch die letztere die weitere Exspiration usf, ausgelöst wäre) liegen allerdings keine vor. - Es muß bemerkt werden, daß Baglioni, dem wir doch so schöne Nachweise der zentralen oder Blut-Beeinflussung der Atemzentren bei den Fischen verdanken, wiederum die von Schoenlein sowie Bethe vertretene Ansicht über den peripheren Ursprung des Atemrhythmus deswegen aufnehmen mußte, da er überhaupt jede Tätigkeit des Zentralnervensystems nur durch periphere Einflüsse zustande kommen läßt. ${ }^{1}$ )

Aber man kann auch für andere Ansichten Stützen anführen. Die rhythmische Tätigkeit der Atemzentren könnte durch innere Reize der schwankenden Blutzusammensetzung hervorgebracht werden, insbesondere durch Anderungen des Sauerstoff- oder Kohlensäuregehaltes im Blute. In dieser Richtung haben Babák und Dèdek das Verhalten des Atemrhythmus der Kiemendeckelbewegungen bei den mit akzessorischen Atemorganen ausgestatteten Fischen gedeutet: wird das Blut von den akzessorischen Atemorganen aus ausreichend mit Sauerstoff versorgt, so wird Apnoë des Kiemenatemmechanismus wahrgenommen, die als eine echte Sauerstoffapnoë aufzufassen ist, während beim Sauerstoffmangel des Blutes, je nach seinem Grade, die apnoischen Pausen sich verkürzen bis sogar ganz verschwinden, und die Kiemenatmung dypnoisch wird. Die Autoren haben den Sauerstoffmangel als den "Auslösungsreiz" des Atemrhythmus angesprochen. (S. auch ihre Versuche an anderen Fischen und die Kohlensäureversuche.) - Baglioni wendet gegenüber dieser Betrachtungsweise ein, daß auch bei entbluteten Tieren und abgetrennten Köpfen rhythmische Atembewegungen sogar stundenlang fortgesetzt werden können. Dieser Einwand scheint uns aber keine Beweiskraft zu besitzen; denn nach der Entfernung der Blutzirkulation kommt im Zentralnervensystem Sauerstoffmangel (und Kohlensäureanhäufung) zustande, wodurch man sogar die zuweilen dyspnoëartigen Atembewegungen der abgetrennten Köpfe usw, erklären könnte. - Die Hypothese der zentral wirkenden Blutreize ist weiter nicht notwendig an das Vorhandensein oszillierender Reiz-

1) Baglioni, S., Zur Analyse der Reflexfunktionen. Wiesbaden 1907. - Die Grundlagen der vergleichenden Physiologie des Nervensystems und der Sinnesorgane. Wintersteins Handb. d. vergl. Physiol. 1910, Bd. IV, S. 1 
wirkungen (behufs Erklärung der rhythmischen Innervationsentladungen) gebunden, denn auch kontinuierlich wirkende Reize können diskontinuierliche Reaktionen auslösen.

Auch eine weitere Theorie bleibt noch diskutabel, obwohl Baglioni meint, daß dafür keine zwingenden Gründe vorliegen. Das sog. Atemzentrum (eigentlich ein komplizierter Nervenmechanismus) könnte eine autochthonne A utomatie besitzen, d. h. eine in ihm selbst, aus seinen eigenen Ernährungsprozessen hervorkommende Automatie. Diese "primäre“ Automatie, die sich auch unabhängig von irgendwelchen sonstigen inneren oder peripheren Beeinflussungen durch rhythmische Innervationsentladungen in die Atemmuskeln kundgeben würde, wird dann chemisch (ev. osmotisch) durch Anderungen des inneren Mediums einerseits (wobei es sich wieder um Folgen der primären Anderungen des äußeren Mediums oder um verschiedene im Körper selbst entstandene Stoffwechseländerungen handeln kann) - andererseits auf den Wegen der Nervenbahnen durch periphere Reize der verschiedensten Art abgeändert ev. reguliert. Es würde sich da um Änderungen der Erregbarkeit der Atemzentren handeln, oder es könnten vielleicht auch spezifische "Atemreize" auftreten, welche den aus den inneren Ernährungsbedingungen resultierenden Stillstand der Atembewegungen unterbrechen würden.

Nach meinen neueren Untersuchungen lassen sich einige bemerkenswerte Umstände zur Beleuchtung der uns interessierenden Probleme anführen.

Zuerst weise ich darauf hin, daB man unabhängig von den Kiemenatembewegungen bei den Embryonen von gewissen Fischen der Genese von speziellen neuen Atemzentren beiwohnen kann $\left(\mathrm{Babak}^{\mathbf{1}}\right)$. Vor dem Funktionieren des Kiemenatemmechanismus, ja sogar lange vor der morphologischen Ausbildung des Kiemenapparates, treten bei gewissen Embryonen sehr ausgiebig tätige rhythmische Flossen- (Haplochilus) oder Schwanzschwingungen (Cichliden) auf, denen die Bedeutung von "provisorischen" Atembewegungen beizulegen ist. Die zugehörigen Atemzentren ändern ihre automatische Tätigkeit nach dem Sauerstoffgehalte des äußeren Mediums; man sieht da, daB z. B. die Schwanzbewegungen der Cichlasoma-, Acara- und anderen Cichliden-Embryonen im sauerstoffreichen Wasser nur kleine Atemperioden, durch lange Pausen getrennt, aufweisen - diese Tätigkeit der Atemzentren könnte man als den Ausdruck der "primären“, autochthonnen Automatie halten. Mit sinkendem Sauerstoffgehalt des äußeren Mediums geht diese Periodizitat in einen ununterbrochenen Atemrhythmus über, bis eine ausgesprochene Intensitäts- und Frequenz-Dyspnoë hervorgebracht wird. Wir haben bei Polimanti ${ }^{2}$ ) eine ähnliche Beobachtung über rbythmische allgemeine Körperbewegungen bei Scylliumembryonen gefunden; es ist nach des Autors Feststellung bemerkenswert, daB der erst später einsetzende Kiemenatemrhythmus mit diesem Rhythmus der allgemeinen Körperbewegungen vollständig übereinstimmt. - Es scheint also, als ob dem

1) Babák, E., Uber die provisorischen Atemmechanismen der Fischembryonen. Zentralbl. f. Physiol, 1911, Bd. XXV, S. 5. - Zur Atmung der Fischembryonen und Jungfische. Blätt. f. Aq.- u. Terr.-Kunde 1912, Bd. XXIII, S. 134.

2) Polimanti, O., Utber den Beginn der Atmung bei den Embryonen von Scyllium (catulus Cuv., canicula L.). Zeitschr. f. Biol. 1912, Bd. LVII, S. 237. 
ganzen 7entralnervensystem eine ursprüngliche Fähigkeit zu den automatischen rhythmischen Bewegungen innewohnen würde, wovon später nur dem Kiemenatmungszentrum eine solche übrig bleibt.

Wir haben weiter das Erscheinen der ersten Kiemenatembewegungen bei den Embryonen von Haplochilen, Cichliden, Forellen beobachtet. Diese ersten Atmungen erscheinen als unregelmäßige Mundöffnungen; der Mund kann da auch längere Zeit offen bleiben. Dann kommen kleine Gruppen von unregelmäBigen, verschieden großen Bewegungen zum Vorschein, durch längere Pausen getrennt. Aus dem allmählich regelmäBig periodisch werdenden Atemrhythmus entwickelt sich endlich ein (mehr oder minder gleichmäßiger) ununterbrochener Typus. Die ersten periodischen Atmungen könnte man als den Ausdruck der „primären“, autochthonen Tätigkeit des herangebildeten Atemzentrums auffassen; durch das sekundäre Beitreten der verschiedenen Regulationsvorgänge kommt dann der ununterbrochene Atemrhythmus zustande.

Aber auch bei erwachsenen Fischen, sogar auch bei solchen, die keine akzessorischen Atemorgane besitzen (durch welche nebst der Kiemenatembowegungen der Gasgehalt des Blutes und demnach auch die Tätigkeit des Kiemenatemzentrums beeinflußt werden könnte), läßt sich ähnliches Verhalten nachweisen, welche uns bei den Embryonen zuerst aufgefallen ist.

Bei Loricaria läßt sich oft, wenn man das ruhige (aber nicht schlafende) Tier beobachtet, besonders, wenn es mit seinem eigentümlich geformten Saugapparat (s. Rauther ${ }^{1}$ ) an der vertikalen Aquarienwand angeheftet ist, ein eigentümlicher Atemrhythmus wahrnehmen. (Er läßt sich sehr schön besonders an der herab- und wieder aufsteigenden Bewegung des Mundbodens verfolgen, welche Bewegung lebhaft an die Kehlatmung der Anuren erinnert. Es empfiehlt sich mittels einer Lupe die Beobachtung durchzuführen, um auch die zuweilen ganz feinen Oszillationen zählen zu können.) Wir sehen da zuweilen vollständige Pausen, die bis über eine Minute dauern können. Nach der Pause erscheinen zuerst sehr feine Mundbodenoszillationen, welche allmählich auffälliger, bis ganz groß, aber wegen der zugleich sehr wachsenden Frequenz kaum zählbar werden, um dann wieder sowohl was die Frequenz als auch die Amplitude betrifft, allmählich abzunehmen, bis die ganz feinen Mundbodenoszillationen völlig verschwinden. Nach einer Pause wiederholt sich die ganze Erscheinung mehr oder minder ähnlich. Zuweilen kommen nur schwache, nicht zählbare Oszillationen als eine größere Periode vor; oder es erscheint nur eine kleine Gruppe von solchen Oszillationen, um in eine Pause überzugehen, wieder eine kleine Gruppe und so fort, bis wieder einmal eine auffällige Atemperiode gezäblt werden kann. - Auch wenn man das Tier stört und zur Schwimmlokomotion zwingt, beobachtet man nach der wiederholten Anheftung sehr rasch das eben geschilderte Verhalten.

Bei demselben Tier wird nun durch sauerstoffarmes Wasser ein maschinenartig regelmäBiger ununterbrochener Atemrhythmus hervorgerufen; bei der gleichen Temperatur steigt bald die Zahl der Atembewegungen immer mehr an (bis über 400 in einer Minute), so daß genaue Zählung un-

1) Rauther, M., Beitrāge zur Kenntnis der Panzerwelse. Zool. Jahrbücher, Abt. f. Anat. u. Ont. d. Tiere 1911, Bd. XXXI, S. 497. 
möglich ist. Dauert der Sauerstoffmangel (im ausgekochten Wasser) länger, z. B. eine halbe Stunde, so verkleinert sich die Atemfrequenz ganz merklich, aber die Exkursionen sind ungemein groß; später beginnt auch die Größe der Atembewegungen mit weiterer Frequenzabnahme herabzusinken. Wenn man da, wenn schon Zeichen der Lähmung erscheinen, frisches Wasser dem Tiere zuführt, erhöht sich bald die Frequenz der Atmung so außerordentlich, daß die Zählung ganz unmöglich wird. Es läßt sich kein günstigeres Objekt zum Nachweis der Dyspnoëerscheinungen sowohl während der Erstickung als auch während der Erholung nach dem Erstickungsversuche denken. (Loricaria eignet sich auch vorzüglich zu den Versuchen über den Einfluß der Belichtung und Verdunkelung, der Erschütterungsreize, der Gewöhnung an dieselben usw., worüber ich später einmal berichten will. Ich bemerke noch, daß Loricaria keine Darmatmung aufweist.)

\section{Summary.}

The author gives a critical review of the opinions that have been pronounced about the origin of the breathing rhythm in the fishes. One cannot accept the theory of the exclusively peripheric origin of the breathing rhythm, though it cannot be doubt that the peripheric stimuli possess a great regulating power over the breathing movements (in the first place the "proprioceptive" stimuli, in the second the centripetal innervations from the mucous membranes etc.) The lack of oxygen in the water becomes by means of the alterations of the blood an important internal factor of the dyspnoic breathing movements of the fishes; a similar action of $\mathrm{CO}_{2}$-accumulation in the blood seems hitherto not to be sufficiently demonstrated. It is possible that the centrum of the breathing movements possess a primary, autochthonic rhythmical activity, based on its own metabolism; the alterations of the internal medium and the complexe reflectory innervations are the main regulators of that primary rhythmical activity. The author gives examples of the ontogenetic evolution of the rhythmical branchial movements in the embryos of Haplochilus, Cichlasoma, Acara, Trutta, of the evolution of other rhythmical breathing-mecanisms ("provisorische Atemmechanismen“) in the embryos of Haplochilus (pectoral fins), Cichlids (caudal breathing rhythm). The adult Loricaria possess in the norm a remarkable periodical breathing rhythm, lack of oxygen in the water causes an other uninterrupted breathing rhythm. 